\title{
A computational method for the computer simulation of the liquid phase sintering of metallic systems
}

\author{
Hamed Hosseinzadeh ${ }^{1, *}$ \\ ${ }^{1}$ Henry M. Rowan College of Engineering, Rowan University, NJ \\ * Corresponding author: Email address: Hamed@uwalumni.com
}

\begin{abstract}
The growth of solid particles during liquid phase sintering was modeled by the Cellular Automata method. The binary phase diagram and Fickian approach for the diffusion process were applied to simulate the chemical composition variation in liquid and solid phases during sintering. The OswaldRipening effect was considered during the dissolution of the solid phase in the liquid phase. It is used to define the probability of solid-phase dissolution by the liquid phase and develop the model to simulate the alloy with solid solubility. So, the microstructure could be modeled in the liquid phase sintering process.
\end{abstract}

Keywords: Metal 3D printing; Computer simulation; Liquid phase sintering; Microstructure; OswaldRipening effect

\section{Introduction}

One of the known densification methods in powder metallurgy is the liquid phase sintering (LPS). It is possible to design some metals $3 \mathrm{D}$ printing based on liquid phase sintering densification. So, it is instructive if there is an efficient computational method to simulate the microstructure of the LPS process at different temperatures. Metal 3D printing is rapid prototyping, and it can be beneficial for producing non-structural applications for design purposes [1]. ASTM Standard F2792 has categorized additive manufacturing processes as Directed Energy Deposition (DED) and Powder Bed Fusion (PBF). Recently, metal 3D printing gains more attention, and the physical and mechanical properties of the printed sample were experimentally studied [2-7]. There are limited examples of 3D printed samples ready for application without additional postprocessing [8,9]. So, most printed samples need additional postprocessing $[10,11]$. There are some computational facilities and models for process modeling of DED and PBF 3D printing methods $[12,13]$. But we do not have a model for considering LPS for the 
metals 3D printing technology. Liquid phase sintering of two metals or alloys could provide some useful manufacturing methods for the metals 3D printing and the 3D printing of metal matrix composite with or without postprocessing.

The presence of the liquid phase in the LPS method is the key parameter that has the role of activation in the diffusion process. In this method, the temperature during sintering is selected between the two elemental melting points of mixed powders. So, the element with a low melting point is melted. It is the first stage of the liquid phase sintering. In the second stage, solid particles are partially dissolved by the produced liquid phase. Solid particles start to dissolve from their higher energy points on their surface. Consequently, the low size particles (higher surface curvature) are thoroughly dissolved in the liquid phase, and it helps the growth of the larger solid particles. It is the so-called Oswald-Ripening effect. The two important parameters of the liquid phase sintering process are the wetting conditions of the solid particles by the liquid phase and the solubility of solid particles into the liquid phase. At a specified temperature, solidification of the liquid phase is the function of the chemical composition of the liquid phase [14].

The growth mechanism and the shape of the solid particles depend on surface energy. Solid particles with anisotropic surface energy (i.e., $W C$ particles in $C o$ liquid) have the preferential growth on the special crystal planes. These kinds of solid particles have spatial geometry with flat surfaces. If the surface energy of the solid particles is isotropic, no preferential crystal planes exist for the growth. The achieved topology of these particles is the non-symmetry sphere [15].

So, the physics of the liquid phase sintering process consists of solidification, dissolution, and diffusion phenomena in the solid or liquid phases. Metallography of the liquid phase sintered samples reveals the channel between the two nearest particles or completely attached surfaces of the two particles. The presence of this channel is related to the penetration of the liquid phase across the two compacted solid particles. The penetration of the liquid phase is a function of the wetting and dihedral angle [16].

Some stochastic models have been developed to study the changing of physical parameters during liquid phase sintering. J. Aldazabal et al. introduced the model using the Monte Carlo method [15]. This model has only considered the precipitation of the solid-phase using the predefined chemical composition for the liquid phase as the initial condition. So, this model cannot be able to simulate the OswaldRipening effect and the formation of the channel between the adjacent solid particles under the use of the wetting and dihedral angles concepts. This model relates the presence of the channel between the two 
solid particles to the restriction of their growth by the chemical composition of the liquid phase. Another stochastic model was introduced by the Po-Liang Liu et al. Their model has focused on the relation of the wetting and dihedral angle to the penetration of liquid phase across the solid particles.

The present computer model has considered the solution of the solid particles in the liquid phase as a stochastic process to simulate the Oswald-Ripening effect, but this model can not able to simulate the penetration of the liquid phase across the two adjacent solid particles. In addition, the pure liquid phase of the low melting point element, which has completely enclosed the solid particles, was assumed as an initial condition. The diffusion in the solid particle was modeled because of its significance to determine the chemical composition at the surface of the solid particles to define the probability of solid-phase dissolution by the liquid phase. It also develops the model to simulate the alloy with solid solubility during sintering. This model uses the same physical dimension and time scale for both the stochastic method and the finite difference method, which are used in solidification/dissolution and diffusion processes, respectively.

\section{Model description}

The differential equation of the diffusion process was solved by the finite difference method. The solidification and dissolution were also modeled using a stochastic method. Both methods need a discretized environment. Each discrete part of the model is called an element. At the center of each element, a node was considered, and the calculation results on each node were extended to its related element as a mean value.

A weighted coordination number was defined for each element. In two dimensional, each element has two kinds of relationships with their neighbor elements. In the first case, two neighbor's elements only contact by their corners and their faces (Fig. 1). The weighted coordination number of the two contacted elements by their corners defines 0.25 , and it also defines 1 when the two neighbor elements contacts by their corners. This weighted coordination number can be used as a representation of the surface energy. If the coordination number of the solid element at the surface of the solid particles increases, the surface energy at this point decreases. The same relation can be used between the coordination number and curvature of the surface. So, the higher surface energy and higher curvature are equal to lower coordination numbers and vise versa. These weighted coordination numbers were used to define the 
probability numbers for dissolution and solidification. Figure 2 shows some possible weighted coordination numbers of a liquid element relate to its adjacent solid elements.

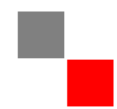

(a) (b)

Figure 1 - Each square shows the element, a) Elements have only the relation by their corners, and b) Elements have contact with their faces.

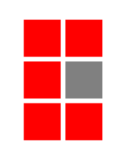

3.5

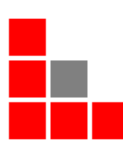

2.75

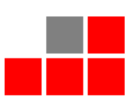

2.5

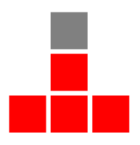

1

1.5

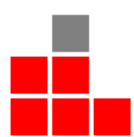

1.25

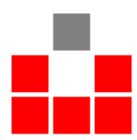

0.5

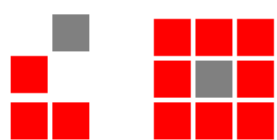

0.25
5

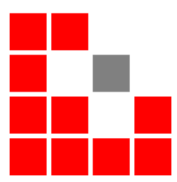

0.75

Figure 2 - Some possible weighted coordination numbers of a liquid element relate to its adjacent solid elements. The red color is related to the solid elements, and the gray one is the liquid element.

Two numbers were defined between 0 and 1 to set up the stochastic concept in the discretized model. The first number is related to the dissolution probability of an element at the surface of the solid particles, and the second number is related to the solidification probability for the liquid elements adjacent to solid elements.

For the diffusion process, the parabolic differential equation can be solved using a finite difference method. The differential equation and its finite difference equation of the diffusion process have been shown in equations 1 and 2, respectively. 


$$
\begin{gathered}
\frac{\partial c}{\partial t}=\alpha\left(\frac{\partial^{2} c}{\partial x^{2}}+\frac{\partial^{2} c}{\partial y^{2}}\right) \\
c_{i, j}^{t+1}=c_{i, j}^{t}+\alpha \frac{\Delta t}{(\Delta x)^{2}}\left[\left(c_{i+1, j}^{t}-2 c_{i, j}^{t}+c_{i-1, j}^{t}\right)\right. \\
\left.+\left(c_{i, j+1}^{t}-2 c_{i, j}^{t}+c_{i, j-1}^{t}\right)\right]
\end{gathered}
$$

Equation 2 is stable if the $\alpha \frac{\Delta t}{(\Delta x)^{2}}$ has the value lower than the 0.5 .

Figure 3 shows the initial physical environment of the model. Each solid particle shown in figure 3 has its own color as its own characteristic. Each new element added to the surface of the solid particle gains this color. So, the boundary between the two neighbor's solid particles can be distinguishable.

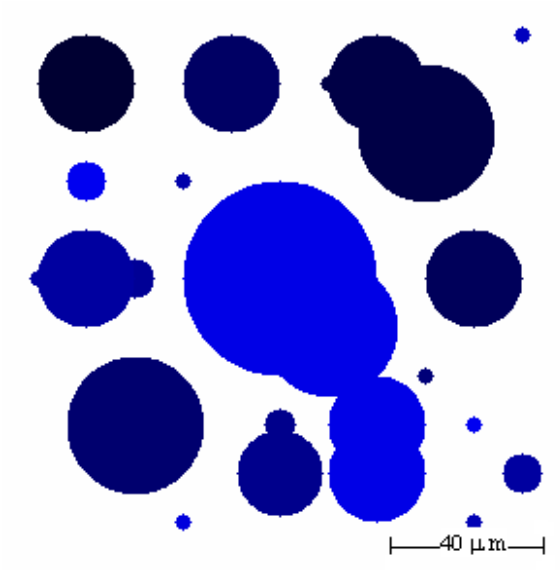

Figure 3 - Simulation environment, blue rounded shapes represent the solid particles, and the white portion shows the liquid phase.

For stochastic calculation, only the surface elements of the solid particles and their adjacent liquid elements attribute to the calculation during each step of the simulation. All the elements must be attributed to the calculation for the diffusion process in solid or in the liquid phase. And the whole model must be discretized. Figure 4 shows the discretized model. The time scale of each step can be calculated by defining the real physical length of each element.

The probability numbers for each physical variable is needed to simulate the solidification or dissolution of an element. A probability number is defined between 0 and 1 to model the solidification of a liquid element. Half of the value of this defined probability number is related to the coordination number, and the rest is related to the chemical composition. During the simulation, a new number is 
randomly generated then compared with that defined probability number. If the value of a randomly generated number is the lower value of the defined probability number, that pixel changes its state.

Each element of the model has four properties. The first is for the distinction of the phase, solid, or liquid. The second is for the chemical composition of an element. The third is for the weighted coordination number, and finally, the fourth is the position of that element.

\section{Result and discussion}

As an example, the $\mathrm{Fe}-\mathrm{Cu}$ system was considered. At the $1300{ }^{\circ} \mathrm{C}$ temperature, it was assumed that the molten $\mathrm{Cu}$ has completely enclosed solid Fe particles. The model has 300 pixels in height and the 300 in its width (Fig. 3). Each pixel, which is square, has the $0.5 \mu \mathrm{m}$ length in width and $0.5 \mu \mathrm{m}$ in height (Fig. 4).

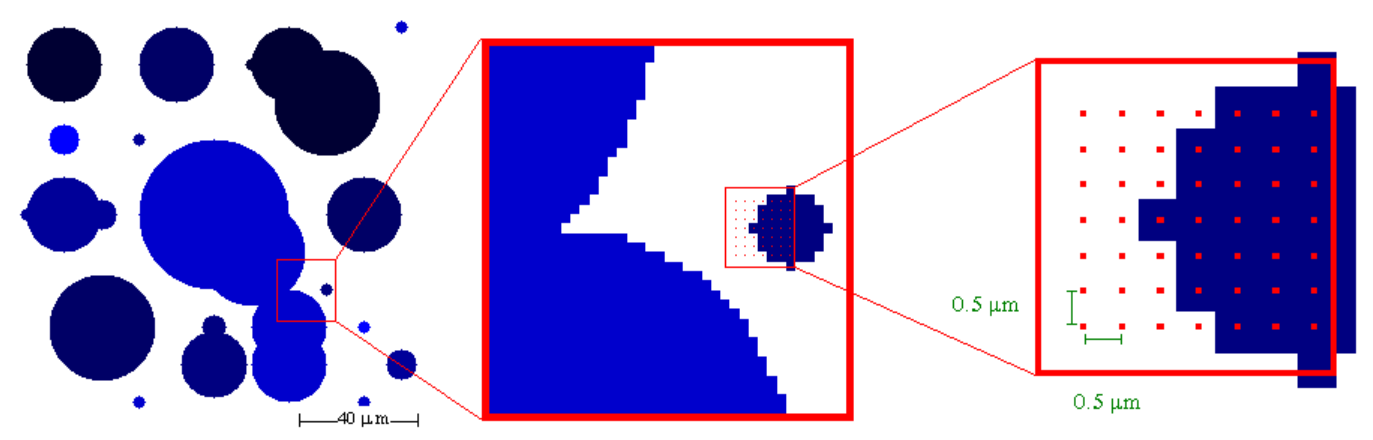

Figure 4 - Discretization of the model. The magnified part of the model shows the uniform meshing and its nodes.

So, the lowest length scale was considered $0.5 \mu \mathrm{m}$ in this simulation. It was assumed that the value of the diffusion coefficient is $0.55 \frac{(\mu m)^{2}}{S}$. It only needs to determine the value lower than 0.5 for $\alpha \frac{\Delta t}{(\Delta x)^{2}}$ in equation 2 to calculate the real-time in each step during the simulation. If 0.01 is considered as the value of $\alpha \frac{\Delta t}{(\Delta x)^{2}}$, then the lowest time scale is equal to 4.5 milliseconds.

$$
\alpha \frac{\Delta t}{(\Delta x)^{2}}=0.55 \frac{0.0045}{0.5^{2}}=0.01
$$

If we assume that the diffusion coefficient is ten times greater than the solid one, then the value of $\alpha \frac{\Delta t}{(\Delta x)^{2}}$ is 0.1 for the liquid phase. It only needs to put 0.1 and 0.01 for the value of $\alpha \frac{\Delta t}{(\Delta x)^{2}}$ equation 2 for liquid and solid phases, respectively. If it is considered that the needed time for $0.5 \mu \mathrm{m} \times 0.5 \mu \mathrm{m}$ area of the solid phase to dissolute is about 4.5 milliseconds, it is logical to consider the same time and length 
scales in both stochastic and diffusion processes. Their numbers of calculation's steps are the same because of the same time and length scales for stochastic and finite difference calculations.

We must define the rule of the stochastic concepts of the solid particles' growth after discretizing the model and defining the length and time scales. Solidification's probability of the liquid element is determined based on its chemical composition and its coordination number. The weight percent of Fe in the liquid was used as the representation of the chemical composition. According to the binary phase diagram of $\mathrm{Fe}-\mathrm{Cu}$, three different conditions can be distinguished for the solidification of a liquid element (Fig. 5).

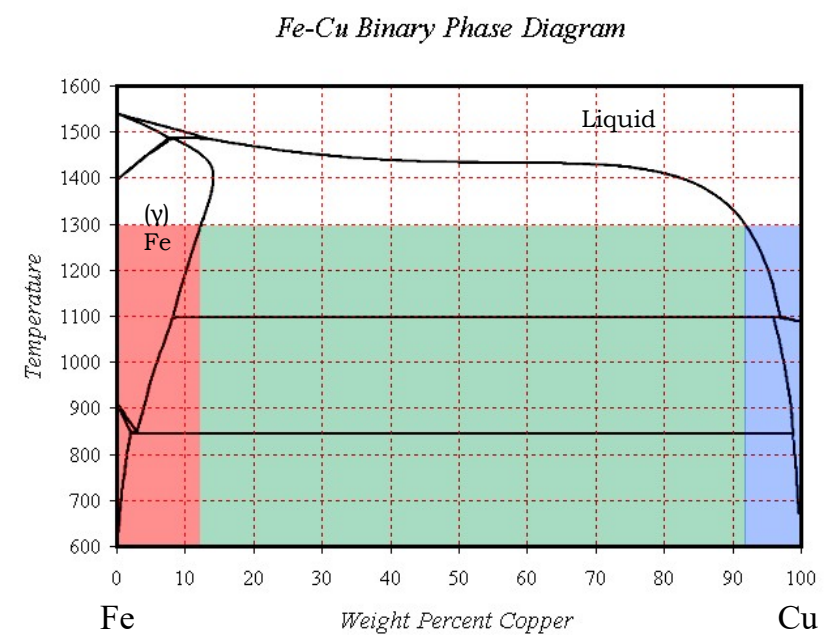

Figure 5 - Binary phase diagram of the $\mathrm{Fe}-\mathrm{Cu}$ system.

First condition: Fe weight percent of a liquid element has a value higher than the $87.5 \mathrm{wt} \%$. Therefore, this element has a chance of solidification.

Second condition: Fe weight percent of a liquid element has a value lower than $87.5 \mathrm{wt} \%$ and higher than $8.5 \mathrm{wt} \%$. In this case, that pixel also has a chance of solidification. But if the pixel solidifies, then the new solidified pixel has 87.5 weight percent of $\mathrm{Fe}$, and the additional new liquid phase is also produced with 8.5 weight percent of Fe according to the phase diagram. This new liquid phase changes the chemical composition of its adjacent liquid elements.

Third condition: Fe weight percent of a liquid element has a value lower than $8.5 \mathrm{wt} \%$. In this case, there is no chance of solidification. So, to solidify this liquid element, some solid elements must be dissolved by the liquid phase to change the weight percent of $\mathrm{Fe}$ in liquid to the permissible range. 
If there is no restriction based on the chemical composition of the liquid phase for solidification, the probability number or chance of the solidification must be defined for each liquid element. First, the solid adjacent pixels of each liquid element must be specified. Then based on table 1 , a number between 0 and 0.5 related to the weighted coordination number will be assigned. The $(-1)$ number was defined for the case of no chance of solidification. The numbers in table 1 are defined based on the best microstructure results by trials and errors.

Table 1 - Relation between the coordination number and the probability number of a liquid element.

\begin{tabular}{cccccccccccc}
\hline Index & $\mathbf{1}$ & $\mathbf{2}$ & $\mathbf{3}$ & $\mathbf{4}$ & $\mathbf{5}$ & $\mathbf{6}$ & $\mathbf{7}$ & $\mathbf{8}$ & $\mathbf{9}$ & $\mathbf{1 0}$ & $\mathbf{1 1}$ \\
\hline Weighted coordinate number & 5 & 4.75 & 4.50 & 4.25 & 4 & 3.75 & 3.50 & 3.25 & 3 & 2.75 & 2.5 \\
Probability number of solidifications & 1 & 0.5 & 0.5 & 0.5 & 0.5 & 0.3 & 0.2 & 0.2 & 0.25 & 0.25 & 0.25 \\
\hline Index & & & & & & & & & \\
\hline & $\mathbf{1 2}$ & $\mathbf{1 3}$ & $\mathbf{1 4}$ & $\mathbf{1 5}$ & $\mathbf{1 6}$ & $\mathbf{1 7}$ & $\mathbf{1 8}$ & $\mathbf{1 9}$ & $\mathbf{2 0}$ & $\mathbf{2 1}$ \\
\hline Weighted coordinate number & 2.25 & 2 & 1.75 & 1.50 & 1.25 & 1 & 0.75 & 0.50 & 0.25 & 0 \\
Probability number of solidifications & 0.009 & -1 & -1 & 0.005 & 0.005 & -1 & -1 & -1 & -1 & -1 \\
\hline
\end{tabular}

We define a new number between 0 and 0.5 according to the chemical composition. The relation of chemical composition number (in the range of $8.5-87.5 \mathrm{Fe}$ wt \%) and the probability number of solidifications is linear in this model. Simply, the final probability number of solidifications for each liquid element is the summation of the two probability numbers (coordination number and chemical composition).

Each solid pixel has a chance of dissolution in the liquid phase if the Fe weight percent is higher than $87.5 \mathrm{wt} \%$. The chance of solution linearly increases with the weight percent of Fe. Similarly, the two probability numbers between 0 and 0.5 were defined for solidification. Then, their summation is used to define the final probability number.

Some simulated results of the $\mathrm{Fe}-\mathrm{Cu}$ system have been shown in figure 6 . The brown color shows the gradient of the Fe weight percent in the liquid phase. This Brown color represents the weight percent of Fe in the range of $8.5-87.5 \mathrm{wt} \%$ in the liquid phase. So, the brown-colored liquid elements have the chance of solidification. The weight percent of Fe lower than $8.5 \mathrm{wt} \%$, which is white, has no chance of solidification. The growth of solid particles during liquid phase sintering is controlled by the diffusion in solid and in liquid phases. 

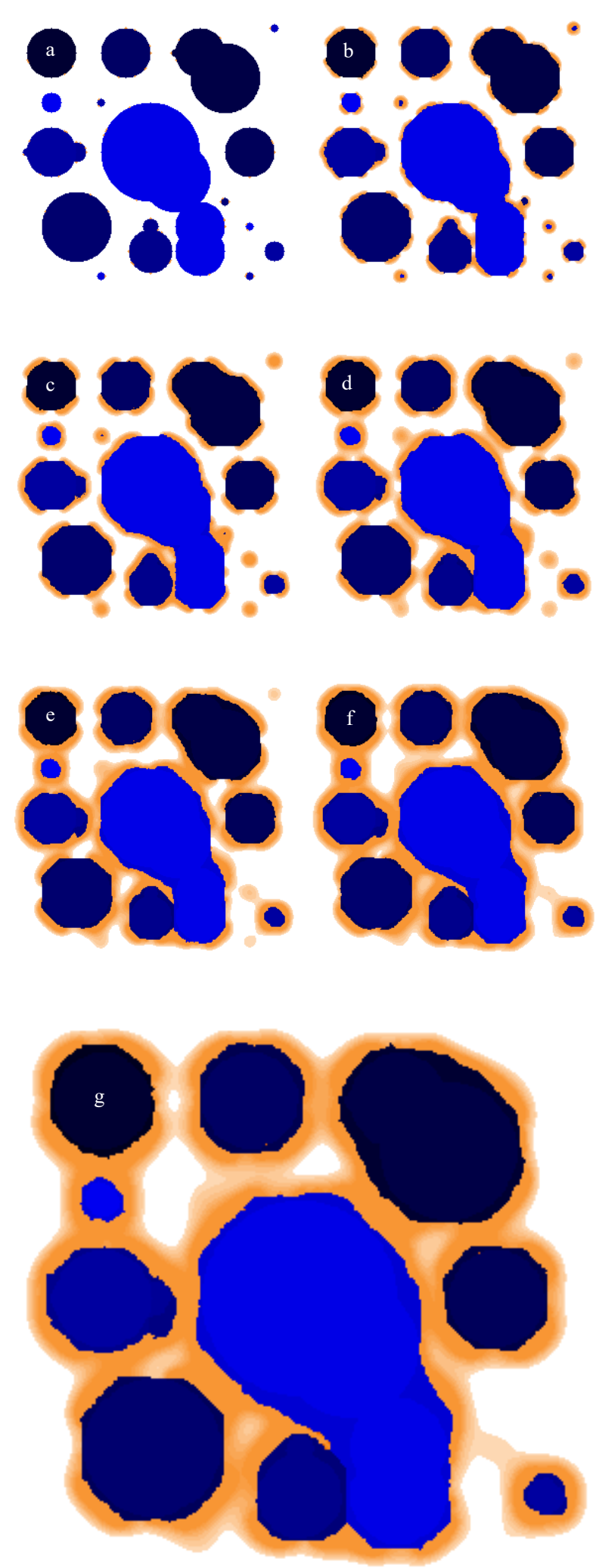

Figure 6 - Simulation results of the $\mathrm{Fe}-\mathrm{Cu}$ system at $1300 \mathrm{C}$.

a) Initial condition, after b) $0.315 \mathrm{~s}$, c) $0.63 \mathrm{~s}$, d) $0.945 \mathrm{~s}$, e) $1.26 \mathrm{~s}$, f) $1.575 \mathrm{~s}$, and g) $1.89 \mathrm{~s}$. It was assumed a virtual binary alloy system with the permissible range of solidification within $50-87.5 \mathrm{Fe} w \mathrm{wt} \%$. 
Considering another permissible chemical range of solidification in a liquid phase shows the change in the reshape speed of solid particles and the solution of the solid element in the liquid phase. The solidification range of the $\mathrm{Fe}-\mathrm{Cu}$ system is $8.5-87.5 \mathrm{wt} \% \mathrm{Fe}$. It was assumed a virtual binary alloy system with the permissible range of solidification within $50-87.5 \mathrm{Fe} w \mathrm{w} \%$. In this case, there is not enough liquid with a proper chemical composition for solidification (brown color), and the growth/reshape's kinetic, or speed of the powders is slower than the case of the real Fe-Cu system. The result has been shown in figure 7 . So, this computational algorithm could provide a simulation facility for engineering the microstructure and compositions of the alloys during phase sintering and make them relevant for metals $3 \mathrm{D}$ printing.
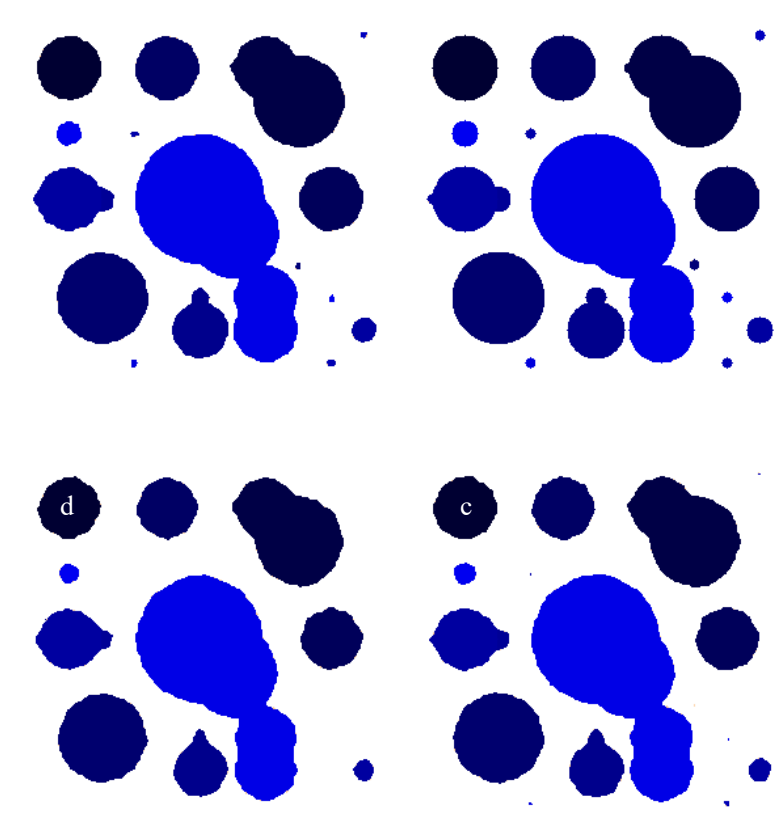

Figure 7- Surface melting of the solid particles rearranges the elements at the surface of the solid particles. a) Initial condition, after b) $0.315 \mathrm{~s}, \mathrm{c}$ ) $0.63 \mathrm{~s}$, and d) $0.945 \mathrm{~s}$. It was assumed a virtual binary alloy system with the permissible range of solidification within $50-87.5 \mathrm{Fe} w \mathrm{w} \%$.

\section{Conclusions}

A computational method was developed for simulating the liquid phase sintering process. Surface dissolution and the effect of Oswald - Ripening of the solid particles were considered in this computer model to achieve the proper microstructure during liquid phase sintering. The model was used to simulate the liquid phase sintering of the $\mathrm{Cu}-\mathrm{Fe}$ system at a constant temperature. This computational code can be extended to the other metals systems and metal matrix composites. This model can be used to study alloys 
with solubility both in the liquid phase and solid one, due to its ability to consider the diffusion process both in solid and liquid phases.

\section{References}

1. Debroy T, Wei HL, Zuback JS, et al (2018) Progress in Materials Science Additive manufacturing of metallic components - Process, structure and properties. 92:112-224

2. Sergio M, Lima F De, Sankaré S (2014) Microstructure and mechanical behavior of laser additive manufactured AISI 316 stainless steel stringers microstructure and mechanical behavior of laser additive manufactured AISI 316 stainless steel stringers. J Mater 55:526-532.

https://doi.org/10.1016/j.matdes.2013.10.016

3. Shi X, Ma S, Liu C, et al (2017) Materials Science \& Engineering A Selective laser melting-wire arc additive manufacturing hybrid fabrication of Ti-6Al-4V alloy : Microstructure and mechanical properties. Mater Sci Eng A 684:196-204. https://doi.org/10.1016/j.msea.2016.12.065

4. Pham MS, Dovgyy B, Hooper PA (2017) Materials Science \& Engineering A Twinning induced plasticity in austenitic stainless steel 316L made by additive manufacturing. Mater Sci Eng A 704:102-111. https://doi.org/10.1016/j.msea.2017.07.082

5. Croteau JR, Grif S, Rossell MD, et al (2018) Acta Materialia Microstructure and mechanical properties of Al-Mg-Zr alloys processed by selective laser melting. 153:35-44. https://doi.org/10.1016/j.actamat.2018.04.053

6. Nassar AR, Reutzel EW (2015) Additive Manufacturing of Ti-6Al-4V Using a Pulsed Laser Beam. Metall Mater Trans A 46A:2781-2789. https://doi.org/10.1007/s11661-015-2838-z

7. Shamsaei N, Yadollahi A, Bian L, Thompson SM (2015) An overview of Direct Laser Deposition for additive manufacturing; Part II: Mechanical behavior, process parameter optimization and control. Addit Manuf 8:12-35. https://doi.org/10.1016/j.addma.2015.07.002

8. Shayesteh Moghaddam N, Saedi S, Amerinatanzi A, et al (2019) Achieving superelasticity in additively manufactured NiTi in compression without post-process heat treatment. Sci Rep 9:111. https://doi.org/10.1038/s41598-018-36641-4

9. Wang YM, Voisin T, McKeown JT, et al (2018) Additively manufactured hierarchical stainless steels with high strength and ductility. Nat Mater 17:63-70. https://doi.org/10.1038/NMAT5021

10. Li J, Cheng X, Li Z, et al (2018) Materials Science \& Engineering A Improving the mechanical 
properties of $\mathrm{Al}-5 \mathrm{Si}-1 \mathrm{Cu}-\mathrm{Mg}$ aluminum alloy produced by laser additive manufacturing with post-process heat treatments. Mater Sci Eng A 735:408-417. https://doi.org/10.1016/j.msea.2018.08.074

11. Zhuo L, Wang Z, Zhang H, et al (2019) Effect of post-process heat treatment on microstructure and properties of selective laser melted AlSi10Mg alloy. Mater Lett 234:196-200. https://doi.org/10.1016/j.matlet.2018.09.109

12. Hosseinzadeh H (2020) Direct laser deposition metal 3D printing at different temperatures of the printer' s chamber : computer simulation. 14-17. https://doi.org/10.20944/preprints202009.0125.v1

13. Hosseinzadeh H (2020) Microstructure and the local mechanical properties of the 3D printed austenitic stainless steel : Computer simulation. Preprints. https://doi.org/10.20944/preprints202008.0573.v1

14. Natali C Di, Poliero T, Sposito M, et al (2019) Design and Evaluation of a Soft Assistive Lower Limb Exoskeleton. https://doi.org/10.1017/S0263574719000067

15. Aldazabal J, Martín-Meizoso A, Martínez-Esnaola JM (2004) Simulation of liquid phase sintering using the Monte Carlo method. Mater Sci Eng A 365:151-155. https://doi.org/10.1016/j.msea.2003.09.021

16. Liu PL (2006) The relation between the distribution of dihedral angles and the wetting angle during liquid phase sintering. Comput Mater Sci 36:468-473. https://doi.org/10.1016/j.commatsci.2005.05.006 